\title{
PROCESOS COLECTIVOS Y PENSAMIENTO ALTERNATIVO EN UN ESCENARIO DE CAMBIO
}

\author{
Alfonso Dubois \\ Miembro del Instituto de Estudios sobre Desarrollo y Cooperación Internacional HEGOA \\ (UPV/EHU)
}

DOI: $10.1387 /$ lan-harremanak.16092

\section{ABSTRACT}

El artículo recoge la intervención hecha en el Seminario ${ }^{1}$ como aportación a su propuesta de tratar de "definir las ideas-fuerza, las claves fundamentales que deberian servir de guía para detectar, visibilizar y priorizar alternativas frente al poder de las empresas transnacionales». Como reacción a ese cuestionamiento se presentan algunas referencias que permitan identificar las preguntas clave que ordenen el debate y señalar los compromisos ineludibles para quienes se esfuerzan por la construcción de la alternativa, que aunque no cubren lo ambicioso del tema, por lo menos no eluden el desafio que plantea.

Palabras clave: alternativa, crisis, complejidad, discurso, acción colectiva.

The article takes up the presentation at the Seminar as a contribution to its purpose of attempting to "define the core ideas, the fundamental keys to serve as a guide to detect, make visible and prioritise alternatives to the power of transnational corporations." As a reaction to this questioning, some references are presented to make it possible to identify the key questions that structure the debate and point out the inescapable commitments for those trying to build an alternative. While these

\footnotetext{
1 Paz con Dignidad y OMAL organizaron el Seminario «Alternativas al poder de las empresas transnacionales. Construcción de un marco teórico», celebrado en Bilbao el 23 de junio de 2015 en el Centro Cultural La Bolsa.
} 
cannot fully encompass a topic as ambitious as this, at least they do not evade the challenge it poses.

Keywords: alternative, crisis, complexity, discourse, collective action.

Artikulu honek Mintegian izandako parte hartzea jasotzen du. Parte hartze horretan proposamena da saiatzea "gida gisa erabili beharko ziren oinarrizko galderak zehaztea, enpresa transnazionalen boterearen aurreko aukerak topatu, ikustarazi eta lehenesteko". Galdera hauei erantzunez erreferentzia batzuk zehazten dira eztabaida antolatzeko eta alternatiba bat eraikitzeko ahaleginak egiten dituztenentzako, ezinbestekoak diren konpromisoak adierazten laguntzeko. Honek ez du hain gai zabala bere osotasunean hartzen baina gutxienez ez dio ihes egiten jarritako desafioari.

Hitz gakoak: alternatiba, krisia, konplexutasuna, diskurtsoa, ekintza kolektiboa. 


\section{Algo hay que cambiar, pero ¿qué?}

Nadie pone en duda que el escenario de nuestro mundo se caracteriza por una serie de procesos de cambio especiales por su alcance y profundidad ${ }^{2}$. En consecuencia, igualmente existe una reacción general que reconoce que el orden económico actual no puede continuar y que entramos en una época de incertidumbre y vulnerabilidad en la que las constricciones de la naturaleza marcarán de forma importante la economía mundial ${ }^{3}$. Estas preocupaciones no son privativas de sectores críticos sino que se encuentran en las instancias representativas de las actuales estructuras de poder, como pueden ser el Fondo Monetario Internacional ${ }^{4}$ o el Foro Económico Mundial5 ${ }^{5}$, el primero como la institución

2 Dos ejemplos entre las muchas formulaciones existentes:

a) Moris (2014) señala trece grandes cambios: el aumento del capital sin patria, la contrarrevolución conservadora de la década de 1980, la implosión de la URSS, el ascenso de China, el surgimiento de los estados BRIC, la urbanización, el aumento de la política identitaria, el resurgimiento de África, el cambio a un estado de no guerra, la creciente amenaza del cambio climático, las experiencias de la primavera árabe, los mundos digitales que amplían y aumentan la velocidad. Estos cambios sugieren un sistema emergente diferente, que tiene profundas diferencias de cómo vemos la economía de la política de nuestro planeta.

b) Bitar (2013), recoge seis tendencias:

c) - Tecnologías disruptivas, aquellas en gestación y cuya difusión transformaría sustancialmente la producción, el empleo, el bienestar, la gobernabilidad y las relaciones humanas.

- Escasez de recursos naturales; cambios en la demanda e innovaciones tecnológicas.

- Transformaciones demográficas, desplazamiento del poder, nuevos mercados, clases medias en ascenso, migraciones.

- Urbanización y expansión de las ciudades, concentración de la población, demanda de infraestructura y servicios básicos, calidad de vida, competitividad de las ciudades.

- Cambio climático, efectos en la agricultura, oportunidades de crecimiento verde, conciencia ciudadana y cambios de comportamiento.

- Gobernabilidad democrática, ciudadanos globales interconectados, impacto de nuevas tecnologías en las relaciones sociales, transparencia, seguridad, violencia y crimen organizado, ciberataques.

3 Kochhar et al. (2014): La economía mundial está atravesando una serie de transformaciones que tornan el futuro considerablemente incierto, complejo e impredecible.

${ }^{4}$ Lagarde, directora del FMI, recientemente hizo un llamamiento para combatir la incertidumbre del futuro global. En:http://www.imf.org/external/pubs/ft/survey/so/2015/new093015a.htm.

5 Más conocido como el Foro de Davos (World Economic Forum) por la reunión anual de personalidades del mundo político y ecónomico, pero que se ha constituido en una institución que pro- 
que asume una función central en la regulación de la economía mundial y el segundo como plataforma de los agentes más significados del capitalismo.

Pero aunque haya consenso en la necesidad de proceder a una revisión, los diagnósticos que se hacen de la situación son muy diversos y, por lo tanto, las exigencias y consecuencias de esa revisión varían de forma importante. La cuestión central es definir la naturaleza del cambio social que afrontamos. Y aquí cabe destacar tres líneas de respuesta entre quienes afirman:

a) estar ante un cambio continuista que el sistema puede asumir con meras adaptaciones;

b) estar ante un cambio que exige modificaciones de las estructuras;

c) estar ante un cambio que exige una propuesta nueva, alternativa.

De acuerdo con ello, la revisión que propone cada uno de los grupos difiere sustancialmente:

- En los dos primeros se propone la revisión de los procesos, las políticas, mientras que los objetivos o prioridades permanecen inalterados o con modificaciones ligeras.

- En el tercero se trata de revisar los propios objetivos del desarrollo y, en consecuencia, también los procesos. Las consecuencias de la revisión que se vislumbra en estas posiciones es muy distinta: en unas el cambio se limita a proponer reformas de funcionamiento del sistema; en la segunda se considera que debe procederse a un replanteamiento de las bases del sistema, se trata de construir una alternativa.

Hay que destacar que se ha producido dentro del primer grupo un transvase de las opciones más reformistas a otras más contemporizadoras. En los últimos años se han matizado de manera significativa las visiones que propugnaban cambios de envergadura, para acabar defendiendo medidas de mero sostenimiento del orden actual acomodándolo a las nuevas condiciones. Las afirmaciones en favor de reformas estructurales profundas que se propugnaban en los primeros momentos de la crisis financiera, se han convertido en un discurso mucho más contenido. Un ejemplo, Martin Wolf, responsable de la línea editorial del Financial Times, en plena ebullición de la crisis en 2009, sostenía opiniones que difícilmente encontrarían hoy acogida en ese diario. Afirmaba que ese era un año decisivo en el que se determinaría el destino de la economía mundial, quizá para generaciones; que quienes mantienen la esperanza de que poder restaurar el crecimiento económico global desequilibrado de la década pasada están equivocados; o que la disyuntiva se encuentra entre una economía mundial más equi-

duce una gran variedad de informes. Ver: http://www.weforum.org/ 
librada y la desintegración; y esta elección no puede posponerse, debe hacerse este ańo... Otro ejemplo lo tenemos en el informe del organismo de Naciones Unidas DESA (2010), que definía los problemas actuales como un momento decisivo de la historia y que para lograr en el futuro una prosperidad a la vez sostenida y generalizada sería necesario introducir reformas considerables en la gobernanza económica mundial y estructurar nuevas ideas en torno al desarrollo económico mundial. Aunque la muestra más representativa de ese cambio se encuentra en los pronunciamientos y agendas de trabajo del G-20. Un breve repaso de las primeras hasta la más reciente reunión pone de manifiesto esta evolución, donde hay que destacar la escasa atención que se les otorga, pasando casi desapercibidas, lo que no deja de ser un indicador de la pérdida de protagonismo ante la falta de trascendencia de sus decisiones y propuestas para la construcción de un nuevo orden.

Pero, al tiempo que esto ocurre en las instancias oficiales, se extienden los diagnósticos que consideran imprescindible una revisión profunda, sobre todo de las ideas necesarias para entender lo que está pasando, lo que se halla en sintonía con la caracterización que propone el Seminario como de crisis civilizatoria, que sitúa la génesis de la crisis en una serie de valores civilizatorios. En esta línea se encuentra Žižek (2012:8) al considerar como premisa básica que el sistema capitalista global se está aproximando a un apocalíptico "punto cero». Los «cuatro jinetes» que empujan a esa situación son: la crisis ecológica, las consecuencias de la revolución biogenética, los desequilibrios dentro del propio sistema y el explosivo crecimiento de las divisiones y exclusiones sociales. Esta percepción de cambio a una nueva era postcapitalista, a la que estamos entrando sin darnos cuenta, es el diagnóstico de Mason (2015), como consecuencia de la tecnología de la información, las nuevas formas de trabajo y la economía de intercambio. Aunque las viejas formas tardarán mucho tiempo en desaparecer, para Mason es el momento de ser utópico. Pronostica que el capitalismo no será suprimido mediante técnicas de marcha forzada sino por la creación de algo más dinámico de lo que existe, al principio, casi invisible dentro del antiguo sistema, pero que se abrirá camino al remodelar la economía alrededor de nuevos valores y comportamientos. A eso le llama postcapitalismo.

Por otra parte, Foster (2014) utiliza el término «crisis epocal» para referirse a la convergencia de unas contradicciones económicas y ecológicas tales que han minado las condiciones materiales de la sociedad en su totalidad, lo que plantea la cuestión de una transición histórica a un nuevo modo de producción. Hoy en día se está produciendo una crisis epocal aún más trascendental que la que produjo la transición del feudalismo al capitalismo, surgida de la expansión ilimitada de un sistema capitalista dirigido al proceso de creación de riqueza abstracta.

En resumen, puede afirmarse que se ha abierto el debate, como no se había producido en las últimas décadas, sobre el futuro deseable y posible que se 
propone tanto para las sociedades locales como para la sociedad global. No se trata de una discusión meramente teórica, sino que es una exigencia para dar respuesta a los nuevos desafíos. Para analizar las distintas propuestas de revisión proponemos como referencia determinar cuáles son los contenidos ineludibles a tener en cuenta y evaluar la respuesta que se da a cada uno de ellos. Entendemos que para ser consecuentes con los retos que se presentan, cualquiera que sea la radicalidad de la respuesta, tiene que abordar las siguientes cuatro dimensiones:

a) Dimensión normativa: hay que preguntarse por el futuro deseable y posible y definir las prioridades en los objetivos a conseguir para las personas y la sociedad ante las nuevas realidades.

b) Dimensión de la naturaleza: estrechamente relacionada con la anterior, exige el replanteamiento de la relación entre los seres humanos y la naturaleza, lo que lleva no solo a reconsiderar los contenidos del bienestar, sino las formas de producción.

c) Dimensión cognitiva: hay que plantear qué nuevas categorías, herramientas teóricas y políticas se necesitan para construir ese futuro, que se presenta complejo e incierto.

d) Dimensión global/local: por una parte, la dimensión planetaria de los desafíos exigen un tratamiento global y, por otra parte, hay que pensar en una nueva articulación de los diferentes niveles de acción política dando un mayor protagonismo al local.

\section{La dimensión normativa}

A diferencia de las décadas pasadas, la ética se convierte en un elemento central e ineludible de la revisión. Y ello porque una cuestión clave del debate actual es la pregunta por el contenido del bienestar, entendido en un sentido amplio. Desde intereses y ámbitos muy distintos surge la necesidad de definir cuál es la vida que merece la pena vivirse. Se trata de establecer qué es estar bien, es decir el bienestar, en este escenario nuevo. En la pregunta se incluye tanto la satisfacción de los objetivos que una persona se marca para su vida como los que una sociedad considera para el conjunto.

La definición del bienestar que se considera valioso corresponde a cada sociedad y será el resultado de un proceso de deliberación colectiva que se produce de diferente forma en cada lugar. Esto no supone desconocer o negar la existencia de elementos comunes y universales sobre las condiciones para que la vida humana sea considerada digna, sino enfatizar la necesidad de que se den procesos participativos de autodefinición, sin condicionamientos e imposiciones externas. 
Desde esta comprensión, la pregunta sobre el bienestar es una cuestión clave en la definición del modelo económico y social que se quiere proponer. Pero las respuestas son muy diversas. Una característica común de la revisión es la toma de conciencia de que el bienestar no puede reducirse a la dimensión material de los recursos. Desde diversas posiciones, han surgido iniciativas de búsqueda de nuevos indicadores ante la creciente convicción de las carencias que sufre la renta per cápita como referencia válida para medir el bienestar de las personas. Este proceso de revisión tiene su importancia ya que supone echar por tierra el indicador del desarrollo por excelencia (el desarrollo entendido no como un término para designar qué hay que hacer con los países en desarrollo, sino en su apreciación más general como es el futuro de cualquier sociedad), indiscutido durante todo el siglo xx. No ha habido ninguna otra referencia que haya gozado de tanta legitimidad para evaluar los resultados del modelo económico. El supuesto era que el incremento de la renta per cápita implicaba el aumento del bienestar general, del progreso o del desarrollo. Plantear la búsqueda de nuevos indicadores supone un cambio que va más allá de una moda o de exigencias coyunturales.

En este proceso de revisión se encuentran las propuestas de la Unión Europea (Más allá del PIB) ${ }^{6}$; de la OECD (Midiendo el progreso) ${ }^{7}$; y del Gobierno francés ${ }^{8}$ o del Gobierno Británico. La que más difusión ha tenido es el Índice para una Vida Mejor, de la OECD, construido a partir de las recomendaciones de la comisión formada por los dos premios Nobel de Economía, Joseph Stiglitz y Amartya Sen, y Jean-Paul Fitoussi, para desarrollar nuevas formas de evaluar el bienestar material y la calidad de vida. El índice integra diversas estadísticas relacionadas con 11 áreas: vivienda, ingresos, empleo, comunidad, educación, medio ambiente, compromiso cívico, salud, satisfacción ante la vida, seguridad y balance entre vida y trabajo (Stiglitz et al., 2013). Estas iniciativas, si bien son interesantes por el reconocimiento de la necesidad de ampliar las dimensiones a tener en cuenta con lo que abren un espacio de debate hasta ahora cerrado para las instancias oficiales, han tenido repercusiones prácticas muy limitadas. Además su alcance se circunscribe a encontrar propuestas de indicadores que permitan recoger mejor la realidad de las vidas de las personas, sin que en esa revisión se pongan en cuestión los fundamentos del modelo económico, sino que se trata de simples reformas de políticas concretas. Pero, en todo caso plantean un campo de confrontación con quienes propugnan revisiones alternativas.

${ }^{6}$ Ver: http://www.europarl.europa.eu/sides/getDoc.do?pubRef=-//EP//TEXT+REPORT+A72011-0175+0+DOC+XML+V0//ES

7 Ver: http://www.oecdbetterlifeindex.org/es/

8 Ver: http://www.stiglitz-sen-fitoussi.fr 


\section{Las revisiones alternativas}

Frente a las iniciativas anteriores, existen otros procesos con la pretensión de trabajar nuevas formulaciones del bienestar, que rompan con las visiones dominantes del desarrollo económico. Hay que destacar el papel que los movimientos sociales juegan en este proceso de redefinición, no solo porque son portadores de las nuevas ideas, sino también actores de su formulación. Resultaría muy prolijo detallar las distintas iniciativas que se encuentran en marcha, pero las fuentes de donde surgen todas ellas pueden agruparse fundamentalmente en cuatro orígenes, que son los siguientes:

a) La nueva mirada a la naturaleza y a las relaciones de los seres humanos con ella. Los graves y crecientes efectos negativos del modelo dominante sobre el planeta —el cambio climático o el agotamiento de los recursos naturales - han llevado a tener que replantear los objetivos del bienestar. La cuestión interesante es que ahora este proceso tiene que considerarse de forma global para todos los países. Se convierte en una pauta obligada de referencia a la hora de establecer los objetivos de bienestar que son realmente posibles sin poner en riesgo la sostenibilidad del planeta como lugar habitable para los seres humanos. Pero son muy distintos los énfasis con que se plantea la revisión. La visión alternativa va más allá de la búsqueda de soluciones tecnológicas que consigan fuentes de energía renovables no contaminantes o de cambios de consumo. Se trata de un cambio de paradigma que afecta al supuesto del crecimiento ilimitado, a la concepción antropocéntrica dominante, a la consideración de la naturaleza como un instrumento, entre otros.

b) Las diferentes visiones del estar bien desde diversas culturas. Las críticas a las concepciones occidentales del desarrollo, que han dominado el panorama internacional, realizadas desde otras culturas encuentran hoy más eco, tanto dentro de los países que las proponen como fuera, aun cuando hace tiempo que venían expresando su profundo desacuerdo con la concepción economicista de la modernización. La visibilidad de los pueblos indígenas y las minorías étnicas como sujetos, objetos y promotores de distintas visiones del desarrollo también ha aumentado dramáticamente y cumplen un papel crítico en aspectos importantes, al denunciar la irracionalidad del desarrollo y la incompatibilidad de muchos proyectos de desarrollo con las cosmovisiones indígenas o al señalar las limitaciones de la euro-modernidad desde la perspectiva indígena. Existe una enorme diversidad de propuestas nacidas desde otras culturas, que se han agrupado en diferentes tendencias, como el postdesarrollismo, el postcolonialismo o el antidesarrollismo. Recientemente ha alcanzado una especial difusión la visión de los pueblos andinos conocida como sumak kawsay o sumak qamaña. Pero no son estas las únicas en 
Latinoamérica, desde la cosmovisión maya hasta las propias de los distintos pueblos indígenas amazónicos hay una enorme variedad de propuestas que obliga a establecer un diálogo entre formas muy diversas de entender el buen vivir?.

c) La economía feminista contiene un cuestionamiento del modelo económico dominante. A nivel teórico las economistas feministas han desarrollado un marco alternativo que va más allá de considerar la exclusión y discriminación de las mujeres y las desigualdades de género. Proponen un marco que conceptualiza el conjunto de la economía, la esfera del mercado, el trabajo pagado y no pagado, la producción y la reproducción social del cuidado. Los conceptos económicos feministas tienen muchos puntos de contacto con las economías morales que se basan en la cooperación, la reciprocidad, se orienta hacia las necesidades y enfatizan la importancia del dar y del cuidado para satisfacer las necesidades.

d) Otras propuestas alternativas de bienestar han surgido de diferentes instituciones académicas o vinculadas con movimientos críticos sociales. Sería muy prolijo recoger todas ellas, existiendo, además, enormes diferencias en cuanto a la fortaleza de sus fundamentos teóricos y a la pretensión de erigirse como referencias universales ${ }^{10}$. Evidentemente, dentro de este apartado hay que encuadrar el enfoque de las capacidades que sirve de base teórica al desarrollo humano.

Es necesario aclarar que el hecho de que una afirmación se encuadre dentro de uno de los anteriores grupos no debe entenderse automáticamente como alternativa. En cada una de esas referencias hay posiciones muy variadas, incluso algunas que pueden calificarse más como continuistas que revisionistas. Pero ello no quita para sostener que desde esas fuentes han aparecido importantes procesos alternativos que ofrecen nuevas visiones del bienestar. Por otra parte, se hace necesario disponer de categorías que permitan establecer puentes de diálogo entre las distintas dimensiones. Al final, la construcción de una nueva vi-

9 Un resumen de las características comunes del buen vivir que propugnan los movimientos sociales andinos:

a) no hay una progresión lineal hacia el futuro, sino un progreso en curso, que siempre se está construyendo;

b) se reconoce a la naturaleza como sujeto;

c) supone vivir en armonía con otros seres humanos y permitir que vivan dignamente y se enfatizan las relaciones de servicio y reciprocidad hacia el otro y la naturaleza;

d) no se separan las dimensiones espiritual y material de la vida;

e) la contextualidad: el buen vivir es específico de cada lugar; y

f) tienen una dimensión utópica (Deneulin, 2012).

10 A modo de ejemplo, dos referencias de propuestas académicas: Stockholm Environment Institute: http://www.sei-international.org/publications?pid=1547); New Economic Foundation: http://www.neweconomics.org. 
sión del bienestar solo podrá darse desde la interacción positiva entre todas ellas. La cuestión es cómo llevar adelante esa tarea.

\section{La dimensión cognitiva}

Una característica del debate es la puesta en cuestión de los presupuestos teóricos y las herramientas de análisis utilizadas por las corrientes hegemónicas de la economía. Los desafíos de una realidad cambiante, que se presenta compleja e incierta, han llevado a amplios sectores del pensamiento económico a revisar los presupuestos teóricos y metodológicos dominantes. Esta deficiencia se destaca al pensar en la economía del desarrollo, cuyos modelos elaborados son representaciones muy simplificadas de una realidad compleja y solo parcialmente entendida. Las teorías del crecimiento que se analizan a través de estudios entre países asumen que todos ellos funcionan igual, sin tener en cuenta los diferentes contextos. Por otra parte, las corrientes hegemónicas de la teoría y la política económica se han dedicado a explicar los factores y causas del crecimiento económico, abandonando la reflexión sobre los conceptos de desarrollo y las teorías del cambio.

En consecuencia, la revisión alcanza también a las formas de conocimiento con las que venimos interpretando la realidad económica y el debate sobre la necesidad de encontrar nuevos enfoques cognitivos aparece como una exigencia para entender los nuevos procesos económicos. Se necesitan categorías nuevas para una narrativa nueva de lo que puede ser el futuro de esta sociedad global de las sociedades locales. La dimensión cognitiva se convierte en un desafío central. Para construir un pensamiento propio que compita con el dominante se requiere asumir el reto de conocer y ello supone una nueva forma de pensar, aprender y practicar un proceso también alternativo de reflexión y análisis. Hay que buscar esforzadamente nuevas herramientas de comprensión.

Para ofrecer una idea de la profundidad y características del reto que se enfrenta, recogemos algunas formulaciones que reflejan diferentes aspectos centrales del mismo. El sociólogo Therborn (2003) enfatiza la necesidad de nuevos procesos de aprendizaje y de búsqueda del conocimiento. Hoy más que nunca, afirma, precisamos una nueva concepción acerca de la transformación social y de la práctica política; una concepción, quizás, posmoderna. Más que una vanguardia que elabore el proyecto «modelo», más que nuevas formas de poder central y concentrado, necesitamos prácticas diferenciadas, flexibles, movimientistas, simultáneamente locales y globales.

Otras propuestas destacan el protagonismo que adquieren hoy facultades antes desechadas por el saber convencional. Para Mason (2015) el poder de la imaginación se convertirá en crítico. 
En una sociedad de la información, ningún pensamiento, debate o sueño se pierde - ya sea concebido en una tienda de campaña, celda de prisión o en el espacio de fútbol de mesa de una compañía de nuevos negocios (startup)—. Necesitamos algo más que un manojo de sueños utópicos y pequeños proyectos horizontales. Necesitamos un proyecto basado en la razón, pruebas y diseños comprobables, que rompe con el "grano" de la historia y es sostenible para el planeta. $\mathrm{Y}$ tenemos que ponernos a trabajar en ello.

El requerimiento de encontrar categorías teóricas idóneas para el escenario actual no viene desde preocupaciones académicas, es la realidad quien las reclama. El poeta francés Paul Valery expresaba muy gráficamente la naturaleza del desafío cognitivo: las ideas se han visto atacadas, sorprendidas y disueltas por los hechos; y somos testigos de algún tipo de insolvencia de la imaginación y de bancarrota de la comprensión. Es el mundo, dice Foster (2014), quien tiene la necesidad urgente de desarrollar el conocimiento de las interconexiones entre el profundo atolladero de la economía capitalista y la amenaza ecológica en rápida aceleración. $\mathrm{O}$, dicho de otra manera, no siempre tenemos las palabras adecuadas para expresar una opinión sobre los problemas nuevos. Por eso, para Taylor (2015) estamos obligados a encontrar el lenguaje que nos permita describir la nueva situación. Vivimos en una era en la que todo cambia muy rápidamente. Necesitamos un lenguaje que dé cuenta de los nuevos significados. Es un proceso sin fin.

\section{La base de la visión alternativa: construcción de espacios comunes}

La mayoría de las revisiones del bienestar se centran en modificar los contenidos del bienestar individual, introduciendo dimensiones inmateriales que pueden abarcar aspectos relacionales como la inclusión en la sociedad, el compromiso cívico y otras, pero obvian la consideración específica del bienestar colectivo. Este abandono de la dimensión colectiva se extiende también en algunos casos a las propuestas alternativas.

El desarrollo humano ofrece una propuesta alternativa de bienestar, que se basa en el enfoque de las capacidades formulado por Sen. Se presenta como un marco normativo, no como una teoría acabada, que establece el espacio a tener en cuenta para asegurar que las personas mejoren la calidad de sus vidas, ya que ellas son los referentes de la propuesta de bienestar. Aquí radica su novedad y su potencialidad, en afirmar que es desde las capacidades de las personas donde puede y debe evaluarse la calidad integral de sus vidas y no en los recursos ni en los resultados materiales. Pero esa atención exclusiva a las capacidades individuales como si ellas agotasen el ámbito del bienestar y fueran su referencia única constituía su principal punto crítico. 
De forma creciente, han surgido posiciones que incluyen la dimensión colectiva como parte integrante del desarrollo humano. Consideran que las capacidades colectivas forman parte del proceso de consecución del bienestar y del bienestar mismo, sin las que el desarrollo humano queda limitado en su potencialidad de crear una alternativa operativa.

Aceptar el marco normativo de las capacidades no significa que se deduzca un contenido único de las capacidades que se consideren prioritarias. No hay una referencia para determinar qué es lo que se considera valioso, sino que cada cultura deberá especificar los criterios con los que realiza esa evaluación. Es decir, caben diversas concepciones del bienestar, más aún necesita de diferentes visiones de la vida. Esto implica la necesidad de que se produzcan procesos colectivos de discernimiento público que vayan definiendo los contenidos de ese bienestar. Un elemento central del enfoque es que cada sociedad o colectivo decide autónomamente sus preferencias o prioridades, necesita de un consenso sobre cuáles son los objetivos para conseguir la vida que estimen valiosa.

No solo desde la legitimación de los objetivos del bienestar surge la necesidad de incluir la dimensión colectiva. El Informe del PNUD (2010), tras veinte años de vigencia del enfoque, revisaba su primera definición, limitada a la consideración del bienestar individual e incluía en la nueva definición:

Participar activamente en el desarrollo sostenible y equitativo del planeta que comparten. Las personas son los beneficiarios e impulsores del desarrollo humano, ya sea como individuos o en grupo.

Los fundamentos del desarrollo humano que ahora se ponen de relieve son su naturaleza sustentable, equitativa y empoderadora y su flexibilidad. No basta con conseguir resultados en un momento dado, ya que estos logros pueden ser frágiles y susceptibles de retrocesos. Más aún, pensando en el derecho de las futuras generaciones, urge velar porque el desarrollo humano perdure en el tiempo, es decir que sea sostenible. El desarrollo humano no es el resultado de esfuerzos individuales, sino que se realiza en el marco de determinadas estructuras que lo impulsan o lo dificulta. Por eso, debe abordar las disparidades estructurales, o sea debe ser equitativo ${ }^{11}$. Además, debe facultar a la gente para ejercer su capacidad de decidir y de participar, dar forma y beneficiarse de los procesos que le competen en el plano personal, comunitario y nacional; es decir, el desarrollo humano debe ser empoderador. Por último, insiste en mantener la deli-

11 El enfoque del desarrollo humano, a partir de sus conceptos centrales de bienestar y de agencia, tiene la posibilidad de cambiar la narrativa del desarrollo a una narrativa de justicia. La cuestión no es tanto si las sociedades son más desarrolladas sino si son más justas o menos injustas y si proveen las condiciones básicas para que las personas vivan bien. El enfoque de capacidades no ofrece herramientas directas para transformar y hacer menos injustas a las situaciones, sino que deja ese proceso a la creatividad de los actores en cada contexto (Deneulin, 2014). 
beración y el debate y dejar siempre la puerta abierta a la discusión. Son las personas quienes, individualmente y en grupo, dan forma a estos procesos.

Estos procesos colectivos se entienden no solo como instrumento, ya que sin su existencia difícilmente las personas podrán conseguir su bienestar, sino como objetivo perseguible por sí mismo, porque los valores y las capacidades colectivas forman parte del bienestar de las personas. Desde esta consideración de la dimensión colectiva, el núcleo constitutivo del desarrollo humano se encuentra en la capacidad de cada sociedad para definir y llevar adelante su futuro, lo que comporta un proceso colectivo de funcionamiento. El desarrollo de una sociedad consiste en el funcionamiento adecuado de todos los agentes involucrados en el proceso común de decisión sobre su futuro.

Afrontar la dimensión colectiva supone, en consecuencia, entrar a conocer el funcionamiento de las instituciones y del sistema en su conjunto. Cuando se hace referencia a las instituciones, se abarca tanto a las formales como las informales. Se trata de entender los procesos de cambio de las instituciones y de sus formas de relación, que constituyen la aventura central de la definición del bienestar y de la estrategia de desarrollo. En definitiva, la capacidad de cambio de una sociedad y de sus instituciones para avanzar en el desarrollo humano.

En definitiva, la propuesta que se hace del bienestar es que el proceso de las dimensiones colectivas de la sociedad es fundamental. No es que se desechen los resultados en las personas, sino que estos solo podrán darse de acuerdo con los valores propios si hay procesos colectivos que los propugnen. En otras palabras, no hay desarrollo si no hay la capacidad de poner en marcha un proceso colectivo. ¿Qué condiciones tienen que darse para que de la interacción entre todas las partes se dé un resultado que permita funcionar como colectivo? El bienestar colectivo implica ese buen funcionamiento y difícilmente se alcanzarán resultados de bienestar colectivo sin la existencia de objetivos comunes. No es posible pensar en capacidades de relación fuertes sin ellos, más bien su calidad y grado de aceptación impulsarán la creación de capacidades colectivas específicas $^{12}$.

Estamos condenados a enfrentar y convivir diferentes visiones del bienestar, distintas valoraciones de lo que es la buena vida, y a buscar plataformas comunes que permitan que esa convivencia sea fructífera y positiva. Todas las culturas pueden aprender de las otras y todas deberán modificar algunos de sus presupuestos de cara a conseguir esa interdependencia inevitable. La historia re-

12 Hay que advertir que cuando se trata de las capacidades colectivas, el tema neurálgico es determinar cuáles son los espacios colectivos de evaluación. En el caso de las capacidades individuales ese espacio alternativo de evaluación ha sido más definido, lo que no quiere decir que se haya cerrado el debate sobre las formas de concretarlo. En el caso de las capacidades colectivas, la determinación de cuales son centrales es objeto de un debate mucho más abierto. 
ciente ha sido una muestra de un comportamiento totalmente contrario: la propuesta de la modernización, en base a los valores occidentales, se hizo hegemónica y ha avasallado a las demás, sin dejar opción al diálogo y a la interacción. La consecuencia ha sido la aniquilación de muchas culturas y haber colocado a otras al borde de la extinción. El siglo xxi ha abierto un nuevo escenario, donde han surgido con fuerza las reivindicaciones de otras visiones del bienestar, de la buena vida, no solo críticas sino a veces directamente contrarias a las prioridades de la modernización.

Hoy no puede admitirse que una sociedad establezca su modo de vida sin referencias a los desafíos globales y sin reconocer su interdependencia con los otros modos de vida. Las consecuencias que ello tiene para las sociedades más ricas, se traduce en la necesidad de revisar las pautas de consumo, insostenibles desde los parámetros actuales. Nos encontramos, pues, ante un proceso complejo y nuevo que pretende crear una sociedad global distinta, que encuentra su riqueza y su dinamismo precisamente en la admisión de la pluralidad de visiones de lo que debe valorarse.

\section{La complejidad del discurso ${ }^{13}$}

Como se ha ido mostrando, el desarrollo, entendido en su sentido amplio como el futuro deseable y posible de las sociedades, tiene como referente el cambio, que se produce en múltiples dimensiones interconectadas entre sí. Nos encontramos frente a un proceso de construcción interactivo entre muchas partes diferentes que, aunque comparten ciertos objetivos comunes, tienen otros objetivos muy distintos y que, incluso, algunas se oponen al cambio social que busca una mayor justicia social. Este proceso se produce, inevitablemente, en el seno de una sociedad estructurada políticamente, por lo que el estado-nación, o cualquier tipo de sociedad local con capacidad de decisión sobre temas comunes se convierten en referencia central del análisis.

La propuesta de un cambio radical requiere considerar el funcionamiento de la sociedad en su conjunto. Ello implica asumir la complejidad como característica del proceso, en cuanto que este tiene como objetivo central la definición del futuro de la sociedad y este no puede quedar en manos de unos pocos o verse sesgada por los intereses de una minoría. Garantizar que ese proceso de definición es realmente participado y fruto de todos los agentes, supone tener que realizar cambios en los roles y responsabilidades de estos, en definitiva supone modificar de alguna manera la estructura de poder.

13 Este apartado se basa en: Alberdi y Dubois (2015). 
El análisis de los procesos de cambio es especialmente complejo. En primer lugar porque se carece de teorías que ofrezcan las herramientas idóneas para analizar el desarrollo del sistema, y, al mismo tiempo, existe una variedad de enfoques parciales que dificultan consensuar el diseño de políticas. En segundo lugar, porque el desarrollo se entiende cada vez más como un proceso incierto, especialmente a partir del abandono de la modernización como modelo a alcanzar. Ya no hay una única referencia sobre cuál debe ser el resultado a aspirar. Ahora el desarrollo se convierte en una aventura que cada sociedad debe emprender sin tener establecidos los objetivos finales ni los caminos por los que deba transitar.

Asumir que la complejidad es una característica central del comportamiento de las sociedades, y específicamente de la economía, abre el debate sobre cuáles son las categorías teóricas idóneas para comprender cómo funcionan y cómo intervenir en ellas. En este sentido, Copestake y Williams (2012) señalan que las prácticas del desarrollo a menudo se representan mejor a través de los llamados «problemas malditos» (wicked), para cuya solución se requiere la colaboración, en lugar de otros problemas que son manejables y pueden solventarse a través de negociaciones o de los considerados críticos que pueden ser objeto de coacción a corto plazo. Tomarse en serio que el cambio de una sociedad incluye un fuerte componente de problemas «malditos» exige que los actores tengan la voluntad de enfrentar cambios más radicales en la forma en que opera como respuesta al análisis. Enfrentar la complejidad implica reconocer la necesidad de colaborar para construir una comprensión más completa del problema.

Por eso, más importante que la propia intervención es el proceso de interacción y descubrimiento. Para ello se requiere un diálogo más abierto y una integración más estrecha de análisis y acción. Esta posiciones, más radicales, reformistas y románticas, implican disponer de modelos mentales alternativos para abordar no un problema de desarrollo individual sino un complejo, incierto y diverso conjunto de problemas de desarrollo inter-relacionados (Copestake y Williams, 2014). Aceptar este planteamiento obliga a funcionar en un marco de aprendizaje para la acción que pone de relieve la importancia de la creatividad, la aceptación de otras culturas y otros ritmos, así como de la reflexividad y la agilidad.

De esta visión de la complejidad se deduce que la forma de afrontar el conocimiento e intervención en esa realidad exige una consideración central de las unidades colectivas locales. La comprensión del funcionamiento de realidades complejas no puede hacerse a través de categorías analíticas pensadas para situaciones sencillas o complicadas, pero inteligibles en su dinámica. Estas resultan inapropiadas para las nuevas realidades. Las nuevas categorías, aquellas que permiten abordar la complejidad, serán fruto de la puesta en marcha de marcos de aprendizaje donde la colaboración, el acercamiento a otras mentalidades, la in- 
teracción entre actores y los consensos o acuerdos básicos que formen parte de un proceso de largo alcance, siempre inacabado. Estas características encuentran mayores probabilidades de concretarse en escenarios de sociedades cohesionadas en base a objetivos comunes. La dimensión local del desarrollo, no en el sentido localista, se convierte en un pilar estratégico para el afrontamiento de la complejidad, si bien no es el único.

\section{Ideas, discurso y acción colectiva}

En respuesta a los enfoques neo-institucionalistas que, a su juicio, no ofrecen un marco satisfactorio para explicar lo que ocurre en nuestros días, Schmidt (2010 y 2011) presenta un marco de análisis que permita captar los procesos actuales de cambio. Este enfoque se ha denominado «institucionalismo discursivo» y bajo ese nombre se agrupa un amplio abanico de enfoques interpretativos de las ciencias sociales que adoptan ideas y discursos que ayudan a explicar las dinámicas del cambio. Es un marco analítico preocupado por el contenido sustantivo de las ideas y los procesos interactivos del discurso en el contexto institucional.

Su planteamiento básico es que las ideas, el discurso y la acción colectiva junto con el análisis de las interrelaciones entre estas categorías van a permitir explicar el conjunto de procesos interactivos a través de los cuales se generan y comunican las ideas. No es un marco para entender el cambio de la sociedad, sino un aspecto concreto y fundamental del mismo: las ideas y los discursos que ayudan a entender las dinámicas de cambio. En un momento caracterizado por un intenso debate sobre el futuro deseable y posible, comprender el proceso de la formación de las ideas y su difusión adquiere un protagonismo especial. En este marco se engarzan los retos de las dimensiones cognitiva y normativa, además de poner de relieve los procesos colectivos de discernimiento. Estamos frente a un escenario de debate donde las ideas importan, pero estas no pueden entenderse fuera del contexto donde se crean y al margen de la preocupación por la acción. Por eso propone un acercamiento endógeno al cambio institucional y una consideración específica de los agentes o actores.

Entendemos que para el análisis de los puntos que hemos venido recogiendo en este artículo, este marco presenta características que lo hacen idóneo para entender y fortalecer los procesos de creación de pensamiento alternativo. Por ello, presentamos un resumen de sus contenidos siguiendo a Schmidt (2011).

El marco se compone de cuatro elementos centrales. En primer lugar, los diferentes niveles a tener en cuenta de las ideas según su generalidad: ideas políticas concretas, ideas programáticas o programas políticos e ideas filosóficas o filosofías públicas. En los tres niveles es necesario que se produzca el cambio de 
ideas, desde los niveles más pragmáticos hasta los más filosóficos que replantean las prioridades del bienestar. El análisis de cuáles son las ideas que se proponen nos servirá para evaluar el tipo de revisión que se lleva a efecto, como se señalaba al principio de este artículo. Los discursos meramente reformistas incidirán las ideas políticas y programáticas, eludiendo las filosóficas. El discurso alternativo deberá formular sus ideas en los tres niveles para poder considerarse como tal.

En segundo lugar, se señalan los contenidos de las ideas. Estas pueden ser cognitivas o normativas, y, lógicamente, tanto unas como otras se encuentran en los diferentes niveles de generalidad señalados, y en diversas formas como: narrativas, marcos, campos discursivos de ideas, prácticas argumentativas, narración de cuentos, memorias colectivas y más. Si bien hemos enfatizado la dimensión normativa por haber sido la más olvidada, igualmente el reto cognitivo se convierte en central, entendiéndolo desde la necesidad de encontrar nuevas categorías y procesos de conocimiento.

Conviene precisar que este énfasis por las ideas no quiere decir que se puede explicar siempre el cambio por medio de las ideas y desde el discurso, ya que los actores actúan a menudo sin tener ideas sobre lo que están haciendo, y se habla de ello cuando ya han actuado. Sin embargo, una vez que las cosas ocurren y los actores actúan, desarrollan ideas y discurso sobre lo ocurrido y lo que hicieron, lo que forma la base de su explicación del cambio, bien sea con ocasión de una crisis o de forma incremental.

En tercer lugar, se encuentran los agentes del cambio político. Las ideas no existen si no se encuentran encarnadas en los agentes. Pero la conexión entre ideas y acción colectiva no es clara. Para este marco del institucionalismo discursivo el vínculo olvidado es precisamente el discurso y la forma en que las ideas transmitidas por los agentes a través del discurso conducen a la acción.

Se destacan dos dominios de la esfera pública donde se producen las interacciones discursivas:

a) el discurso coordinativo entre actores comprometidos en crear, deliberar, argumentar, negociar y llegar a acuerdos; y

b) el discurso comunicativo entre actores políticos y el público comprometido en presentar, contestar, deliberar y legitimar estas ideas. En el primero lo central es la construcción política de las ideas, que requiere la confrontación, la negociación, el diálogo, etc. Supone reconocer nuevas formas de producción de las ideas, concediendo a la participación de personas y grupos un papel central. En el segundo, las ideas políticas, en cualquiera de los tres niveles, se someten a la legitimación de la sociedad que requiere procesos de deliberación y contestación. Las ideas que no se comunican son fugaces e intrascendentes. 
Un aspecto sugerente de este marco es su consideración de los agentes de cambio como agentes conscientes que generan y deliberan sobre ideas a través de interacciones discursivas que llevan a la acción colectiva. Pensar, decir y hacer son de igual importancia para explicar las fuerzas que llevan al cambio político. Las dos esferas, coordinativa y comunicativa, se hallan interconectadas tanto por los contenidos sustantivos como por los procesos interactivos del discurso. Pero no siempre conectan ambos discursos, a veces el discurso permanece centrado dentro de las élites. Los movimientos sociales son fuerzas significativas del discurso comunicativo de abajo hacia arriba.

En cuarto lugar, el contexto del cambio. El contexto institucional importa: las instituciones constituyen el escenario en el que los agentes conscientes son los agentes pensantes que desarrollan sus ideas para la acción que transmiten a través del discurso. El escenario institucional constituye tanto lo que estructura las ideas, el discurso y las acciones de los agentes como lo que se construye por las ideas, el discurso y las acciones de los agentes. Esta tensión e interacción entre ideas y contexto es central para entender las dinámicas del cambio.

\section{Para concluir}

Quienes pretenden el cambio necesitan ideas, modelos y visiones de paradigmas y prácticas alternativas que se hallen basadas en otros principios de los capitalistas y neoliberales. Necesitan disponer de conceptos y elaborar estrategias que alumbren políticas para un pensamiento económico heterodoxo. No hay un modelo finalista, pero tiene que haber un diseño que ofrezca referencias: más de principios que de políticas concretas. Sin diseños previos del futuro que se vislumbra como deseable y posible, no será posible iniciar el camino. En las páginas previas hemos planteado algunas referencias centrales sobre cómo afrontar esta búsqueda.

Este es un proceso de largo plazo y de aprendizaje continuo, que, por encima de todo, debe ser creativo. Creatividad exigida desde el inicio porque enfrentar una realidad compleja e incierta implica saber navegar en la incertidumbre y navegar en común. El primer paso es el reconocimiento de la nueva realidad en la que tenemos que saber desenvolvernos. Eso supone una cultura que no se basa en la certeza sino en la búsqueda de la respuesta a un mundo inacabado que hoy necesita de un especial esfuerzo colectivo para hacerlo humano y habitable. 


\section{Bibliografía}

ALBERDI, Jokin y DUBOIS, Alfonso (2015): «El proceso de definición colectiva del bienestar, como referencia clave de la dimensión política del desarrollo. Del desarrollismo al desarrollo humano", en Engelken-Jorge, Marcos, Cortina, Mercè y Bergantiños, Noemí (eds.), Contextos y perspectivas de la democracia: ensayos en honor a Pedro Ibarra Güell, Bilbao, Parte Hartuz, UPV/EHU, pp. 62-85.

BITAR, Sergio (2013): Las tendencias mundiales y el futuro de América Latina, Santiago de Chile, CEPAL, Inter-American Dialogue. En: http://www.prospectivayestrategia.cl/ pdf/tendencias.pdf

COPESTAKE, James and WILLIAMS, Richard (2012): The Evolving Art of Political Economy Analysis: Unlocking its practical potential through a more interactive approach. Development Futures Paper, Oxford, Oxford Policy Management. En: http://www. opml.co.uk/sites/opml/files/OPM_DF_political\%20economy\%20analysis.pdf

COPESTAKE, James, y WILLIAMS, Richard (2014): «Political-Economy Analysis, Aid Effectiveness and the Art of Development Management», Development Policy Review, 32 (1), 133-154.

DELEUNIN, Séverine (2012): Justice and deliberation about the good life: The contribution of Latin American buen vivir social movements to the idea of justice, Bath Papers in International Development and Well-being n. ${ }^{\circ}$ 17, The Centre for Development Studies University of Bath. En: http://www.bath.ac.uk/cds/

DELEUNIN, Séverine (2014): Wellbeing, Justice and Development Ethics, Londres, Routledge.

DESA (2010): Estudio Económico y Social Mundial 2010. En: http://www.un.org/es/mdg/ summit2010/pdf/overview_sp.pdf

KOCHLAR, Kalpana; SUN, Yan; TSOUNTA, Evridiki y WESTELIUS, Niklas (2014): «La bola de cristal», Finanzas \& Desarrollo, septiembre de 2014.

FOSTER, John Bellamy (2014): "La crisis epocal», Mientras Tanto N.o 125, junio 2014. http://www.mientrastanto.org/boletin-125/ensayo/la-crisis-epocal

MASON, Paul (2015): «The end of capitalism has begun», The Guardian, July 17. http:// www.theguardian.com/books/2015/jul/17/postcapitalism-end-of-capitalism-begun

MORIS, Jon (2014): Reimagining Development 3.0 for a Changing Planet, IDS Working Paper 435, Institute of Development Studies.

PNUD (2010): Informe sobre Desarrollo Humano, 2010, Programa de Naciones Unidas para el Desarrollo, ONU, Nueva York. En: http://hdr.undp.org/es/

SCHMIDT, Vivien A. (2010a): «Taking Ideas and Discourse Seriously: Explaining Change through Discursive Institutionalism as the Fourth New Institutionalism,» European Political Science Review 2(1), 1-25.

SCHMIDT, Vivien A. (2010b): Speaking of change: why discourse is key to the dynamics of policy transformation, Keynote Paper prepared for presentation for the 5 th International Conference in Interpretive Policy Analysis, Grenoble 23-25, junio 2010.

SCHMIDT, Vivien (2011): «A Discursive institutionalism: scope, dynamics, and philosophical underpinnings», en Fischer, Frank and Forester, John (eds.), The Argumentative Turn Revised: Public Policy as Communicative Practice, Durham, NC, Duke University Press.

STIGLITZ, Joseph E., SEN, Amartya y FITOUSSI, Jean Paul (2013): Medir nuestras vidas. Las limitaciones del PIB como indicador de progreso, Barcelona, RBA. 
TAYLOR, Charles (2015): «Las personas no tienen claro hoy el sentido de la vida», El Pais, 8 agosto 2015.

THERBORN, Göran (2003): "La crisis y el futuro del capitalismo», en Sader, Emir (comp.) y Gentili, Pablo (comp.), La trama del neoliberalismo. Mercado, crisis y exclusión social, 2. ${ }^{a}$ ed., Buenos Aires, Argentina, CLACSO, p. 192. http:// bibliotecavirtual.clacso.org.ar/ar/libros/trama/ther.rtf

ŽIŽEK, Slavoj (2012): Viviendo en el final de los tiempos, Madrid, Ediciones Akal. 